\title{
Team-Track: A tool for investigating tracking performance in teams
}

\author{
FLORIAN JENTSCH, CLINT BOWERS, DAN COMPTON, \\ GUILLERMO NAVARRO, and TAMARA TAIT \\ University of Central Florida, Orlando, Florida
}

\begin{abstract}
Team performance and coordination have gained considerable interest in disparate fields (e.g., aviation, process control, medicine, and wartime operations). Team-Track, a $\mathrm{C}$ program that allows controlled experimental manipulation of team conditions, was developed to meet the research needs resulting from this interest. Using Team-Track, one can manipulate task interdependence, team staffing, relative contributions of team members, communications, and informational displays in a low-cost environment. The dependent measure of performance generated by the program is the absolute tracking error along each dimension. The program is ideal for investigating team interactions as it allows for orthogonal manipulation of team variables, therefore accommodating a multitude of research paradigms.
\end{abstract}

The importance of teams is increasing in business, research, and production. Specifically, complex environments with many interdependent operators, such as aviation, surgery, and chemical or nuclear processing, require effective cooperation between individuals. There is a need to investigate and understand the interactions among team members and to use this knowledge to form and train effective teams.

To study the variables that may affect team performance, it is important to create tasks that can adequately tap the coordination and task resources that are required in team operations. This paper describes a low-cost software program for the investigation of these and related factors.

\section{Research Approaches}

In the past, a variety of tasks has been used to study teams and their performance. Approaches ranged in fidelity from realistic field studies to high-fidelity tasks in expensive simulators to artificial laboratory experiments. One problem facing the latter type of laboratory research has been that the validity of artificial tasks and the generalizability of their findings have been questioned (see Johannsen, 1993). On the other hand, there are few guidelines for the execution of team studies in the literature (Johannsen, Levis, \& Stassen, 1994). In fact, Driskell and Salas (1992) pointed out that laboratory research may be superior to studies in realistic settings when investigating teams. They reasoned that laboratory experimentation is

The authors gratefully acknowledge the help of the following individuals in this research project: Curt Braun, Marci Bruce, and Christina Secrease. The authors would also like to express their gratitude to Kimberly Smith-Jentsch and two anonymous reviewers for their comments on earlier versions of this manuscript. All authors were affiliated with the Department of Psychology at the University of Central Florida. Dan Compton is now with Essex Corporation, Orlando. Correspondence should be addressed to F. Jentsch, Team Performance Laboratory, Department of Psychology, University of Central Florida, Orlando, FL 32816-1390 (e-mail: fgj43539@pegasus.cc.ucf.edu). the only approach that allows the degree of control that is necessary to attribute cause-and-effect relationships under the conditions of large variability that characterize team interactions.

On the basis of this notion, previous laboratory investigations into team performance and interaction have employed a number of artificial tasks that ranged in complexity from very simple paper-and-pencil scenarios (e.g., the "balloon problem" in which participants have to make team decisions regarding which items to drop and which to retain) to complicated computer-supported systems, such as the synthetic work methodology (Alluisi, 1967; Morgan \& Alluisi, 1972; Weaver, Bowers, Salas, \& CannonBowers, 1995). However, the large majority of existing team tasks have been dedicated to cognitively oriented problems, such as decision making and resource allocation. There remains a need for research tools to investigate other important team behaviors.

One such area to be investigated includes team-based tracking behavior. Tracking tasks require continuous monitoring and manual control at a variety of workload and difficulty levels. Tracking is an important factor in a large number of real-world applications in which the continuous, coordinated, and goal-oriented application of control inputs is necessary (e.g., transportation, process control, national defense). In many of these areas, teams of human operators have to track the system state and react through the coordinated application of control inputs. Tracking therefore contains many task and process characteristics that are typical of team interactions. Consider, for example, the operation of a submarine: Only the controlled and combined input from several team members (e.g., helmsmen, rudder plane operators, and ballast tank controllers) ensures that the vessel maintains the desired depth and course in rapidly changing environmental conditions.

In addition to the ecological validity even of very simple tracking tasks, using this class of tasks for the study of team behaviors can be particularly useful, since previ- 
ous research into individual tracking performance has provided effective models that allow us to model the influence of task characteristics, such as difficulty, duration, and time pressure on task performance (see Adams, 1989; Johannsen, 1993; Wickens, 1992). In this respect, tracking may be superior to other classes of tasks because it allows us to remove the variance accounted for by the individual task characteristics that moderate larger team processes. As such, tracking tasks allow the researcher not only to closely simulate specific real-world behaviors in the laboratory but also to test general theories about the real world in a controlled setting.

\section{Statement of Purpose}

To initiate a research program into the effects of various aspects of team performance on team tracking, we decided to develop a methodology based on a computerized task. Three main goals were set. First, the hardware and software should allow the systematic and controlled manipulation of several independent variables (IVs) that may affect team performance (e.g., team staffing and composition) while being flexible enough to allow future applications. Second, the system should possess the sensitivity to detect differences in team performance brought about by the manipulation of these IVs. This goal may appear intuitive in any behavioral research, but it often becomes an elusive concept in team studies because of the large increases in variability that occur when team interactions are considered. Third, the setup should be inexpensive to allow its use in a variety of settings.

In response to these goals, we developed Team-Track, an inexpensive $\mathrm{C}$-based program that can be executed on any IBM PC compatible using DOS 3.3 or higher. TeamTrack allows for the systematic manipulation of several variables and the assessment of their effects on team performance in a tracking task. In particular, the researcher can manipulate five factors using Team-Track: interdependence, team staffing, relative contribution of team members, communication within teams, and information display.

\section{TRACKING TASK}

Team-Track consists of a basic two-dimensional pursuit tracking task (Figure 1). One target moves along a horizontal axis, whereas the other moves vertically. For each dimension, a subject-controlled cursor can be moved along a parallel axis, offset by about $25 \mathrm{~mm}$ when presented on 14-in. diagonal monitors. The cursors can be manipulated individually or together. For example, in a dyadic condition with interdependent control, each cursor is controlled by a separate input device (e.g., the vertical cursor is moved through inputs from a joystick, and the horizontal cursor is controlled via a mouse). Through the modular setup of the task, other combinations are possible. Potential combinations also include the control of both cursors through only one input device or the interchange of control devices.

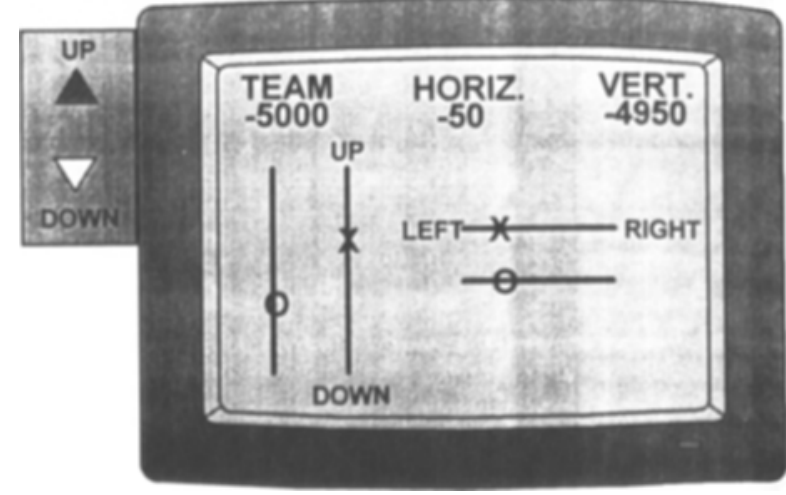

Figure 1. Representation of the display layout. Each target is indicated by an $O$; each participant-controlled cursor is indicated by an $X$. The movement-direction indicator (switchbox) for the control of the vertical cursor is shown in the upper left-hand corner.

The tracking task itself can be manipulated with respect to four variables: difficulty (probability and degree of target movement), duration, composition of input devices (mouse, joystick, etc.), and control characteristics of the input devices (i.e., sensitivity and control-response ratio). Due to its flexible design, the task can also be used as a typical pursuit tracking task with single operators.

The absolute error between the target and the cursor position serves as the dependent variable in Team-Track. It is sampled at least once every second along each dimension, depending upon the computer clock speed. The errors along each dimension are then added over the duration of an experimental session, which can last anywhere from a few seconds to several hours. The sum of the absolute tracking error is usually used as the final dependent measure of performance. The real-time deflection between the cursor and the target can also be presented on the screen, together with a numerical team score value whose scaling can be defined by the experimenter.

\section{MANIPULATION OF TEAM VARIABLES USING TEAM-TRACK}

A number of variables have been hypothesized to affect or moderate the processes within teams and their performance. Process variables, such as the restriction of communications between team members, and task- and team-related variables, such as structure, have been found to affect each other as well as outcome measures of team performance. These variables have been incorporated into a number of team performance models (e.g., Salas, Dickinson, Converse, \& Tannenbaum, 1992). Yet, while the theoretical relationships among these variables are fairly well conceptualized, results from empirical studies that systematically studied their interactions are still lacking.

Toward this end, Team-Track allows the controlled and systematic manipulation of five of these variables: inter- 
dependence, team staffing, relative contribution of team members, communication within teams, and information display. Each variable can be orthogonally controlled, resulting in a large number of possible fully factorial models. Some examples of final models are shown in Table 1.

\section{Interdependence}

Saavedra, Earley, and VanDyne (1993) developed a taxonomy describing the various classes of interdependencies within teams by evoking the construct of complex interdependence (CI). According to Saavedra et al., CI has three components: task interdependence, goal interdependence, and feedback interdependence. The following section contains the definitions of these components and describes how Team-Track can be used to manipulate and study their various levels.

Task interdependence describes the degree to which individual team members need to interact to perform the team's task(s). It can be manipulated in four levels: pooled, sequential, reciprocal, or team interdependence.

1. Under pooled interdependence, each person performs his/her own task, and the team result is the sum of each person's output. With respect to the tracking task, pooled interdependence is achieved when each cursor is controlled individually and independently.

2. Sequential interdependence occurs when one person's output is necessary for another person's input (i.e., B cannot act without output from A). In Team-Track, one or more persons give steering commands that are translated into control inputs by other team members.

3. Under reciprocal interdependence, one person's output becomes another person's input, and vice versa: $B$ depends on $A$, and $A$ depends on B. Using Team-Track, this condition can be achieved in a variety of settings. In a dyadic setup, for example, one person's steering commands become another person's input for the cursor movement, and vice versa.

4. Team interdependence is the highest form of coordinated activity. According to Saavedra et al. (1993), "group members jointly diagnose, problem solve, and collaborate to complete a task" (p. 63). This form of task interdependence is the most difficult to emulate in a laboratory setting. However, in Team-Track, it can be achieved by allowing team members to jointly decide how to best complete the tracking task within the situational constraints. Three-person teams, for example, may decide to have two of their team members give steering commands to the remaining one, who exercises the commands. Alternatively, they may instead decide to have one member give steering commands to the two others, who each control one of the cursors.

Goal interdependence is easily manipulated by providing the team members with a priori goals. These goals can vary between individual goals ("minimize your error score alone") and team goals ("try to minimize the team's error score"). In large teams, combinations of team and individual goals can be issued, allowing an even larger range of conditions to be tested.

Feedback interdependence in Team-Track can be manipulated by limiting the knowledge of results or changing the focus of feedback. Showing only the team's concurrent score but not the individual scores, for example, can be used as a way of achieving team feedback interdependence. Each individual's contribution becomes incorporated in the team score, thus limiting individual feedback. Conversely, when only individual scores are shown, this may limit the utility of feedback with respect to the performance of the team as a whole. Team-Track thus allows the experimenter to study various directions and the quality of feedback.

\section{Team Staffing and Composition}

Team-Track allows the manipulation of team staffing and composition in several levels: $U p$ to five team members can be included in the setup, and responsibilities can be systematically varied. Variations in team staffing can be achieved by limiting the access to information and increasing the number of interactions among team

Table 1

Examples of Possible Research Models Using Team-Track

\begin{tabular}{|c|c|c|c|c|c|c|}
\hline \multirow[b]{2}{*}{ Model } & \multirow{2}{*}{$\begin{array}{c}\text { Team } \\
\text { Staffing }\end{array}$} & \multicolumn{3}{|c|}{ Interdependence } & \multirow{2}{*}{$\begin{array}{c}\text { Relative } \\
\text { Contribution }\end{array}$} & \multirow{2}{*}{$\begin{array}{l}\text { Communication } \\
\text { Within the Team }\end{array}$} \\
\hline & & Task & Goal & Feedback & & \\
\hline A & 2 & Pooled & Team & Indiv./Team & $50-50$ & Verbal \\
\hline B & 2 & Sequential & Team & Indiv./Team & 100 & Signal \\
\hline $\mathrm{C}$ & 2 & Reciprocal & Team & Indiv./Team & $50-50$ & Signal \\
\hline $\mathrm{D}$ & 2 & Reciprocal & Team & Indiv./Team & $20-80$ & Signal \\
\hline $\mathrm{E}$ & 3 & Sequential + Pooled & Team & Indiv./Team & $50-50$ & Signal \\
\hline $\mathrm{F}$ & 3 & Sequential & Team & Indiv./Team & $50-50$ & Signal \\
\hline $\mathrm{G}$ & $2+2$ & Pooled + Sequential & Team & Indiv./Team & $50-50$ & Signal + Voice \\
\hline
\end{tabular}

Note-Explanation of the models: (A) Each team member independently tracks one target. (B) One team member gives the steering commands to the other, who controls both cursors. (C) Team member A controls cursor A but sees only target B; team member B controls cursor B but sees only target A. Team member A gives steering commands to team member B, and vice versa. (D) Same as Model C, but one team member's contribution is weighted more than the other's. (E) Two team members independently give steering commands to one team member, who combines the-information and controls both cursors. (F) One team member gives independent steering commands to two team members, who exercise the commands. (G) Two team members each form a subteam that controls one of the cursors each. 
members: A four-person setup with a combination of sequential and pooled task interdependence can, for example, be realized by limiting the information about target and cursor location to two people (e.g., A and C) who have to give steering commands to the other two people (B and $D$ ). Thus, $B$ and $D$ are dependent upon commands from $A$ and $C$ (sequential interdependence), while the output of the team is the combination of the tracking performance by $A$ and $B$ on one side and $C$ and $D$ on the other (pooled interdependence). Likewise, triadic and dyadic setups can be achieved by changing the responsibilities for the control and physical manipulation of the cursors. Finally, five-person setups can be realized by adding a fifth person, who assigns the tracking commands to the team members, for example, on the basis of their performance.

\section{Team Member Importance}

Team-Track allows the manipulation of the relative amount that each team member can maximally contribute to the team task. In the baseline setting, each target-cursor system contributes equally $(50 \%-50 \%)$ to the team performance score. Any other combination (e.g., 80\%-20\%) is possible by changing the relative contribution of each dimension. Thus, it is possible to investigate whether goal setting and feedback effects are moderated by the perceived importance of team members. One such area in which these effects may be pronounced is that of social loafing (Hardy \& Latané, 1986).

\section{Team Communications}

Through experimental instructions, it is possible to manipulate the communication processes within teams when performing Team-Track. Limiting the communication channels allows the experimenter to investigate whether certain forms of communication tend to increase team performance while other communication methods tend to reduce it. For example, in previous experiments using Team-Track, we prohibited verbal communication between team members (Jentsch, Navarro, Braun, \& Bowers, 1994) and, by restricting the view among team members, removed the possibility of using certain forms of nonverbal communication (e.g., gestures). Instead, the participants were required to use special signal switches to indicate the direction their partners had to move their cursors. In other settings, hypotheses about other forms of communications (e.g., voice, nonverbal gestures) may be under investigation. The use of these channels could then be allowed.

\section{Information Display}

Finally, a small number of display variables can be manipulated with Team-Track. For example, information can be shown or hidden by cardboard overlays that are placed over the VDT screens. The overlays can be used to create task interdependence by limiting access to the targets or change the feedback interdependence by selectively displaying results or limiting access to them. Also, using video splitters, the same or (through the use of overlays) different information can be shown to different team members. Finally, other display variables that can be manipulated include the display lag, update rate, and size, color, and location of displayed information.

\section{PROGRAM DESCRIPTION}

\section{Tracking Routine}

The program was developed under Borland C for DOSbased machines. It utilizes graphics routines particular to the Borland compiler as well as public domain mouse and joystick routines from the Snippets archive maintained by Bob Stout. ${ }^{1}$ The program should be executable on any IBM-compatible computer with at least an EGA video card and 128K RAM free. Mice should be Microsoftcompatible, and joystick input is expected via a standard game port.

The basic operation of the program is (1) attempt to access the file where data is logged (e.g., trackl.dat), (2) detect if a mouse is present, (3) get the session-control values from the configuration file (track.cfg), (4) if the values are invalid, run the configuration routine and save the updated values to the configuration file, (5) initialize the random number generator and set up the graphics mode, (6) run and $\log$ data for each trial in the session to the data file (e.g., track1.dat), and (7) free up system resources and exit. Within each session, the task components for each cycle can be broken down as follows: (1) quit if the maximum number of trials has been run, (2) reset values to their defaults and record the trial starting time, (3) if the current time exceeds the time allotted for this trial, go to Step 10, (4) check mouse and joystick positions and move the cursor accordingly, (5) update the target position, (6) calculate the distance between the cursor and the target along both the $x$ - and $y$-axes, (7) scale these differences based upon the maximum scale and horizontal/vertical components, (8) clear and output current data to a second virtual screen (for smoother video updating), (9) swap the current visible virtual screen with the one containing the current data, and (10) when the current trial is over, display the final scores, $\log$ them to a file, and prepare for the next trial (go to Step 1).

Target movement. The likelihood that a target will move during a cycle is determined by comparing a userselected target movement probability with a random value. If it has been determined that a movement should occur, the actual movement magnitude is calculated as a random value between 0 and the maximum target movement specified by the operator. A separate random number is used for this calculation. To achieve unique random numbers for each trial, the program's random number generator is seeded with a value based upon the date/time values stored in the computer's clock when the program begins. The "playing field" in which the target and cursor can move is limited to 200 units. Target movements are limited to the field boundaries.

Cursor movement. Cursor movement along each axis is currently limited to 8 units (out of 200) per cycle. Deflections via the mouse are determined by the magnitude of mouse movements combined with the current mouse 
sensitivity settings. Joystick movements, on the other hand, deflect the cursor either 4 or 8 units. The maximum cursor movement occurs if the joystick is deflected at least $95 \%$ of its maximum (the limits are determined during joystick calibration and saved in the configuration file). Joystick movements smaller than $95 \%$ of the maximum deflect the cursor only half of its maximum per cycle. The maximum scale or deflection allowed is approximately 64,000 (default is 10,000 ). When calculating the scores, differences in the 200-unit playing field are scaled to match the maximum deflection.

Session control. Values that define session characteristics are contained in the configuration file (track.cfg). The following variables may be modified within certain constraints using any standard text editor: Mice and joystick type and sensitivity, session duration, target movement probability, individual contribution of the vertical and horizontal tracking components, and number of trials in a session. Valid settings for mice and joysticks are particular to the hardware used, as well as to the drivers that accompany them. The duration for each session is limited to approximately $18.2 \mathrm{~h}$ (default at $5 \mathrm{~min}$ ). The target movement probability can be selected by the user between $0 \%$ and $100 \%$; the default is $20 \%$. Likewise, the user can select the individual contributions of each dimension to the combined tracking error as percentages of total. In the default setting, both dimensions contribute equally to the error score $(50-50)$; other settings reflecting unequal task contributions (e.g., 80-20 or 20-80) are possible. Finally, the maximum number of trials that may be run during one session is approximately 64,000 (default is 10 ).

\section{Data Collection}

Currently, the dependent variable collected during each trial is the sum of the tracking errors, sampled approximately once a second for each target and added over the duration of the trial. The error scores for each dimension and the combined team error score are shown on the top of the screen and updated once a second.

\section{Other Features}

Team-Track allows the continuous collection of data for a specified number of trials. In the manual mode, the operator/experimenter has to confirm each new trial before it begins; this allows the experimenter to accommodate for intertask breaks and for pauses in data collection (e.g., when a subject is not ready for the next trial). In the automatic mode, the next trial is started automatically after a certain threshold value (time between trials or number of keyboard presses) is exceeded. The automatic feature allows for easier collection of data when the paradigm calls for no interaction with the participants.

\section{TESTING AND APPLICATION}

\section{Reliability Assessment}

The reliability of the software and hardware was assessed in a large number of automated trials (more than
600 in each instance) on various computers with different input devices and task setups. Consistently, the distributions for the vertical and horizontal error scores did not differ significantly from a normal distribution (see Jentsch, Navarro, \& Bowers, 1994). The results from these trials indicated that the computer program did not introduce systematic errors in the tracking task.

\section{Team Performance Research}

We first used Team-Track in a research program that investigated the effects of varying feedback on performance in team tasks. On the basis of previous research (e.g., Briggs \& Johnston, 1967), we had hypothesized that team members who had to perform two tasks simultaneously would optimize that task for which they received feedback and neglect the other. We tested this hypothesis in several experiments that systematically varied the feedback interdependence of tasks within the framework of complex interdependence (Saavedra et al., 1993). That is, we used Team-Track to test hypotheses about feedback effects under various conditions of task interdependence and team staffing. The results are briefly summarized below, but they are described in more detail elsewhere (i.e., Jentsch, Navarro, \& Bowers, 1994; Jentsch, Navarro, Braun, \& Bowers, 1994; Jentsch, Tait, Navarro, \& Bowers, 1995).

Results from dyads. In the first setup that we used in our experimental program, dyads performed Team-Track under reciprocal task interdependence and were given team goals: Each team member gave the other member steering commands on one dimension and simultaneously exercised the other team member's steering commands in the other dimension. Two studies were performed: one using a within-subjects paradigm (Jentsch, Navarro, Braun, \& Bowers, 1994), and the other using a mixed between-/within-subjects design (Jentsch, Navarro, \& Bowers, 1994). Both studies had essentially the same results: When receiving feedback on performance along the dimension they controlled, the team members receiving feedback tended to neglect the task of giving their partners steering commands (i.e., the team aspects of the tasks). Likewise, when feedback was provided on the team performance (i.e., considering the tracking performance of the other team member), performance improved on that aspect, whereas it decreased on exercising the commands from the partner. Thus, we found experimental evidence for our hypothesis that feedback provided on one task aspect would lead to tradeoffs in that team members emphasized the task aspect for which they received feedback while neglecting other tasks.

Results from triads. On the basis of our findings from the study of dyads, we concluded that the potential negative effects of feedback (i.e., the tradeoffs among tasks) occurred because team members had to balance two tasks and feedback diverted their attention onto a single task aspect. In light of this, we theorized that only teams in which team members had to perform two important tasks simultaneously could be affected. Conversely, we hypothesized that the teams that had no competing tasks 
should not be adversely affected by feedback for only one task.

We tested this hypothesis using two groups of triads with different internal structures: In one group, one team member had to control both cursors; in the other, the control of the cursors was split among team members (see Jentsch et al., 1995, for a detailed description). In short, the results mostly confirmed our hypothesis: Teams in which one member had to control both cursors showed the characteristic performance tradeoffs we had previously seen in dyads. When control of the two cursors was split among two team members, however, these tradeoffs were not present. Instead, the pattern of means suggested that performance along both dimensions could improve even under partial feedback, perhaps because it freed resources within the team that could be used to improve overall performance.

Demonstrations using four-person teams. To demonstrate the feasibility of using Team-Track with larger teams, we conducted a small pilot study using four-person teams. In the demonstration setup, two team members each formed two subteams consisting of one "controller" and one "helmsperson." Each subteam was charged with the control of one target dimension, but the whole team was asked to perform as best they could together, even if that involved helping each other out. Although, for the purposes of this demonstration, only a small number of teams completed the task, observations confirmed that the simulation showed typical processes demonstrated by work groups and teams (e.g., team self-correction). Also, we saw evidence for the emergence of a team leader, indicating the importance of leadership in team settings.

\section{Summary}

The experiments and demonstrations we conducted with Team-Track consistently showed that tracking performance was affected by the manipulation of feedback interdependence. As hypothesized, team members in various team settings appeared to focus their attention onto those tasks for which they received feedback and tended to neglect other tasks. Furthermore, the experiments confirmed that the specificity of feedback can be important for its effectiveness: The relatively unspecific "team" feedback did not provide clear information to the teams as to the process they could use to improve their overall performance.

Additionally, we observed that Team-Track was easy to learn by our research participants. The students appeared to grasp the principles and mechanics of Team-Track within a very short time: Usually, three 2-min practice trials were sufficient. This observation was supported by the experimental results that consistently did not show any systematic practice effects over 9-12 experimental trials.

\section{SUMMARY AND OUTLOOK}

Team-Track is a program that allows the controlled manipulation of team variables in a variety of interdependent team situations. It fulfills the three goals that we set in response to the need for more research into team behaviors.

1. The first of these goals was that the software and hardware should allow the systematic and controlled manipulation of team variables. As we demonstrated, TeamTrack allows the easy and cost-effective manipulation of variables, such as team interdependence, staffing, communication, and information display. It is therefore possible to create many fully factorial models that accommodate a large proportion of research questions.

2. Team-Track has successfully demonstrated its sensitivity to detect differences in team performance in the feedback research program described above. This indicates that the tracking task is capable of eliciting strong changes in team processes with relatively simple manipulations of team variables.

Additionally, the results from our experiments showed that Team-Track can be used not only to simulate very specific tasks and behaviors in the laboratory but also to test general theories about team behaviors in a controlled environment (i.e., the construct of complex interdependence; Saavedra et al., 1993). Repeatedly, under different experimental setups, the hypotheses suggested by the theoretical framework correctly predicted the results observed in the simulation. As a result, we believe that TeamTrack can be a useful tool for the study and testing of general team performance theories.

3. Cost reduction was the final goal that governed the development of the software. It was achieved through the use of public-domain, off-the-shelf program components and by incorporating the software in an established hardware configuration. Also, features that would have increased software and hardware cost were not programmed but were manipulated through the use of low-cost alternatives. For example, the display field limitations for the various feedback conditions were manipulated via cardboard overlays, rather than by programming separate software routines. Also, communication restrictions between team members were achieved through the use of switchboxes (compare Figure 1). This greatly reduced cost when compared with the use of additional input/output devices (e.g., touchscreens) and their associated routines. The cost of the experimental setup (excluding computer and monitor) was kept at a minimum: Expenditures in material reached only $\$ 25$, and assembly and testing required about $10 \mathrm{~h}$.

Throughout the development, modularity of the design was paramount. Flexible changes and additions to the design can be made without the high cost of complete system redesign. In the future, several additional features can be incorporated by adding short segments of code, increasing the number of possible team research applications even further.

\section{HARDWARE REQUIREMENTS AND PROGRAM AVAILABILITY}

Team-Track should be executable on any IBM-ATcompatible computer with at least an EGA video card 
and 128K RAM free, DOS Version 3.3 or greater. Mice should be Microsoft-compatible, and joystick input is expected via a standard game port. Persons interested in obtaining a copy of Team-Track can do so by sending a formatted 3.5-in. floppy disk and a stamped, self-addressed envelope to F. Jentsch, Team Performance Lab, Department of Psychology, University of Central Florida, Orlando, FL 32816-1390, or by sending an e-mail request for electronic transmission of the program to the lead author at fgj43539@pegasus.cc.ucf.edu.

\section{REFERENCES}

ADAMS, J. A. (1989). Human factors engineering. New York: Macmillan. ALLUisl, E. A. (1967). Methodology in the use of synthetic tasks to assess complex performance. Human Factors, 9, 374-384.

Briggs, G. E., \& Johnston, W. A. (1967). Team training (Rep. No. NAVTRADEVCEN-1327-4). Orlando, FL: Naval Training Device Center.

Driskell, J. E., \& Salas, E. (1992). Can you study real teams in contrived settings? The value of small group research to understand teams. In R. W. Swezey \& E. Salas (Eds.), Teams. Their training and performance (pp. 101-126). Norwood, NJ: Ablex.

Hardy, C., \& LatanÉ, B. (1986). Social loafing on a cheering task. Social Science, 71, 165-172.

Jentsch, F., Navarro, G., \& Bowers, C. (1994). Tradeoffs in a team tracking task as a function of performance feedback. In Proceedings of the Human Factors and Ergonomics Society 38 th Annual Meeting. October 24-28, 1994, Nashville, TN (pp. 1204-1208). Santa Monica, CA: Human Factors and Ergonomics Society.

Jentsch, F., Navarro, G., Braun, C., \& Bowers, C. (1994). Automated feedback in teams: Trading individual for team performance. In C. James \& M. Salzman (Eds.), Proceedings of the 2nd Mid-
Atlantic Human Factors Conference, Washington, D.C., February 23 26, 1994 (pp. 69-73). Washington, DC: George Mason University. Jentsch, F., Tait, T., Navarro, G., \& Bowers, C. (1995, October). Differential effects of feedback as a function of task distribution in teams. In Proceedings of the Human Factors and Ergonomics Society 39th Annual Meeting (pp. 1273-1277). Santa Monica, CA: Human Factors and Ergonomics Society.

Johannsen, G. (1993). Mensch-Maschine-Systeme [Human-machine systems]. Berlin: Springer-Verlag.

Johannsen, G., Levis, A. H., \& Stassen, H. G. (1994). Theoretical problems in man-machine systems and their experimental validation. Automatica, 30, 217-231.

Morgan, B. B., \& AlLuisi, E. A. (1972). Synthetic work: Methodology for the assessment of human performance. Perceptual \& Motor Skills, 35, 835-845

SaAvedra, R., Earley, P. C., \& VanDyne, L. (1993). Complex interdependence in task-performing groups. Journal of Applied Psychology, 78, 61-72.

Salas, E., Dickinson, T., Converse, S., \& Tannenbaum, S. (1992). Toward an understanding of team performance and training. In R. Swezey \& E. Salas (Eds.), Teams: Their training and performance (pp. 3-29). Norwood, NJ: Ablex.

Weaver, J. L., Bowers, C. A., Salas, E., \& Cannon-Bowers, J. A. (1995). Networked simulations: New paradigms for team performance research. Behavior Research Methods, Instruments, \& Computers, 27, 12-24.

WICKENS, C. D. (1992). Engineering psychology and human performance (2nd ed.). New York: Harper Collins.

\section{NOTE}

1. Bob Stout's Snippets archive is located at the FTP site: oak.oakland. edu: /pub/msdos/c.

(Manuscript received August 29, 1994; revision accepted for publication June 20,1995.) 